\title{
Testing Structure Formation in the Universe via Coupled Matter Fluids
}

G. O. Okeng'o ${ }^{1,2}$, J. O. Malo ${ }^{1}$, J. B. Awuor ${ }^{1}$, R. O. Genga ${ }^{1}$

${ }^{1}$ Department of Physics University of Nairobi P. O. Box 30197-00100, Nairobi-Kenya

${ }^{2}$ The Astrophysics Group, Department of Physics University of the Western Cape, P. O. Box Private Bag X17 Bellville-South Africa

\begin{abstract}
We present results from a new framework in which the matter fluid is split into a strongly clustered "halo" component and a weakly clustered "free" component accreted by the haloes. The interaction is modelled using a simple function of the matter density that mimics recently published results from halo theory of $N$-body simulations. We find that whereas the background dynamics are consistent with the standard non-interacting case, the density perturbations are highly sensitive to the energy-momentum transfer, with our model approaching the standard ACDM cosmology today.
\end{abstract}

\section{Introduction}

In the standard cosmological paradigm, the presently observed structures that dominate our Universe are known to have formed from tiny fluctuations in the primordial cosmological fluid that grew under gravitational amplification to form the galaxies, clusters of galaxies and voids observed today (Padmanabhan, 1991; Dodelson, 2003). In spite of the impressive progress made over the last decade, powered by increased observations and computing power, understanding the way in which structures such as galaxies form, still remains one of the most challenging problems in modern cosmology today that is the subject of some of the planned next generation large surveys such as the Square Kilometre Array (SKA) (Padmanabhan, 1991).

The standard cosmological model- also called the $\Lambda$-cold dark matter (or $\Lambda \mathrm{CDM}$ )- which is based on Einstein's theory of general relativity and the standard model of particle physics, remains the simplest and most successful model in explaining a wide range of current observational data with a minimum number of free parameters (Durrer \& Maartens, 2008). The prediction by the $\Lambda$ CDM model that the universe is dominated by two "dark" components- dark matter (DM) and dark energy (DE)- that contribute about 26\% and 69\% respectively to the total cosmic energy budget, raises key questions about the nature of the "dark-sector" and large-scale structure formation (Planck Collaboration XVI, 2014).

Motivated by a recently published work by Angulo \& White, 2009) 
that presents the only detailed study of the full mass halo assembly histories in a standard $\Lambda \mathrm{CDM}$ cosmology and the need to explore extensions to the $\Lambda \mathrm{CDM}$ model that can be confronted with data from upcoming large cosmological surveys, in this paper, we explore a new framework in which we split the matter fluid into two interacting components via energy-momentum exchange. This paper is arranged as follows: The general governing background and perturbation equations are given in section 1. In section 2, we introduce our model for the energy-momentum transfer and present key equations. Section 3 provides a solution of the dynamical equations for the interacting scenario. Our discussion of the results and conclusions are given in section 4 .

\section{The governing equations}

\subsection{Background dynamics}

An interaction in the background is described by the general energy conservation equations (Valviita et al. 2008, Clemson et al. 2012),

$$
\bar{\rho}^{\prime}{ }_{A}+3 H \quad \rho_{A}=a \bar{Q} Q_{A}
$$

where $\rho$ are the background A-fluid densities $(\mathrm{A}=\mathrm{h}$, f where " $\mathrm{h}$ " denotes the halo fluid and "f" the free fluid), $H=a^{\prime} a^{-1}$ is the Hubble parameter, $a$ is the scale factor and $\bar{Q}_{A}$ are the A-fluid background energy transfer rates. The "prime" denotes the conformal time derivative. The Hubble parameter evolves according to the Friedmann equation,

$$
H^{\prime}=-\frac{4 \pi G}{3} a^{2} \rho
$$

Here $G$ is Newton's constant and $\Lambda$ is the cosmological constant. In order to solve the background problem, a choice for the energy transfer rate $\bar{Q}_{A}$ is required. This can be achieved by defining the fraction of matter inside halos as a function of the density,

$$
F(\rho)=\frac{\rho_{h}}{\rho_{M}}
$$


where $F(\rho)$ and the densities are perturbed quantities. In the background, it can be shown that the energy transfer rates become,

$$
\bar{Q}_{h}=\frac{\bar{F}^{\prime}}{\bar{F}}=-\bar{Q}_{f}
$$

Hence from (1) we obtain,

$$
\begin{array}{ll}
\overline{\mathrm{\rho}}_{h}{ }_{h}+3 H & \overline{\mathrm{\rho}}_{h}=\frac{F^{\prime}}{F} \bar{\rho}_{h} \\
\mathrm{\rho}^{\prime}{ }_{f}+3 H & \mathrm{\rho}_{f}=-\frac{F^{\prime}}{F}-\mathrm{\rho}_{h}
\end{array}
$$

Which together with (2) yields a complete background solution once a form for $F$ is chosen.

\subsection{The Perturbations}

The perturbed line element for scalar perturbations in the Newtonian gauge has the form Clemson et al. 2012,

$$
d s^{2}=a^{2}\left[-(1+2) d \eta^{2}+(1-2) \delta_{i j} d x^{i} d x^{j}\right]
$$

The interaction leads to an energy-momentum balance given by,

$$
\nabla_{v} T_{A}^{\mu v}=Q_{A}^{\mu}
$$

With $Q_{h}^{\mu}=-Q_{f}^{\mu}$ and $T_{A \vee}^{\mu}$ being the energy-momentum tensor given by (for perfect fluid neglecting anisotropic stresses),

$$
T_{A v}^{\mu}=\left(\rho_{A}+P_{A}\right) u_{A}^{\mu} u_{v}^{A}+P_{A} \delta_{v}^{\mu}
$$

And the A-fluid four velocity is defined by, 


$$
\begin{aligned}
& u_{A}^{\mu}=a^{-1}\left(1-, \partial^{i} v_{A}\right) \\
& u_{\mu}^{A}=a\left(-1-, \partial_{i} v_{A}\right)
\end{aligned}
$$

Here $Q_{A}^{\mu}$ are the energy-momentum four vectors split parallel to the total four-velocity $u^{\mu}=a^{-1}\left(1-, \partial^{i} v\right)$ as,

$$
\begin{gathered}
Q_{A}^{\mu}=Q_{A u}^{\mu}+F_{A}^{\mu} \\
Q_{A}=\bar{Q}_{A}+\delta Q_{A} \\
u_{\mu} F_{A}^{\mu}=0
\end{gathered}
$$

Where $Q_{A}$ and $F_{A}^{\mu}$ are the energy density and momentum density transfer rates along $u^{\mu}$ respectively such that

$$
F_{A}^{\mu}=a^{-1}\left(0, \partial^{i} f_{A}\right)
$$

with $f_{A}$ being the intrinsic momentum transfer potential.

The components of the energy-momentum 4-vectors $Q_{A}^{\mu}$ are,

$$
\begin{gathered}
Q_{0}^{A}=-a\left[Q_{A}(1+)+\delta Q_{A}\right] \\
Q_{i}^{A}=a \partial_{i}\left[f_{A}+Q_{A}(v+B)\right]
\end{gathered}
$$

For each A-fluid (7) yields the density and velocity perturbation equations,

$$
\begin{gathered}
\delta_{A}^{\prime}=\frac{\bar{Q}_{A}}{\bar{\rho}_{A}}\left(\phi-\delta_{A}\right)+\frac{\delta Q_{A}}{\bar{\rho}_{A}}+k^{2} \mathrm{u}_{A}+3 \phi^{\prime} \\
\mathrm{u}^{\prime}{ }_{A}=\frac{\bar{Q}_{A}}{\bar{p}_{A}}\left(\mathrm{u}_{M}-\mathrm{u}_{A}\right)^{-H \quad \mathrm{u}_{A}-\phi}
\end{gathered}
$$

where $v_{A}$ and $v_{M}$ are the A-fluid and total matter peculiar velocities respectively and $\phi$ is the gravitational potential which evolves according to the equation, 


$$
\phi^{\prime}+H \quad \phi=-\frac{3}{2} H^{2} \Omega_{M} v_{M}
$$

\section{The Interacting two-matter fluid model}

\subsection{Background interaction}

For each A-fluid, the background energy transfer rate $\bar{Q}_{A}$ in (4) can be modelled for any physical choice of $F$. Motivated by halo theory N-body simulation results from Angulo \& White (2009) that compute the full CDM halo assembly histories starting from the freestreaming mass scale up to the scale of galaxy clusters, we model the fraction of matter inside halos using three functions that mimics the results by Padmanabhan (1991) for spherical collapse. Figure 3.1 shows fits for our three models for $F$ and the spherical collapse model results in Angulo \& White (2009).

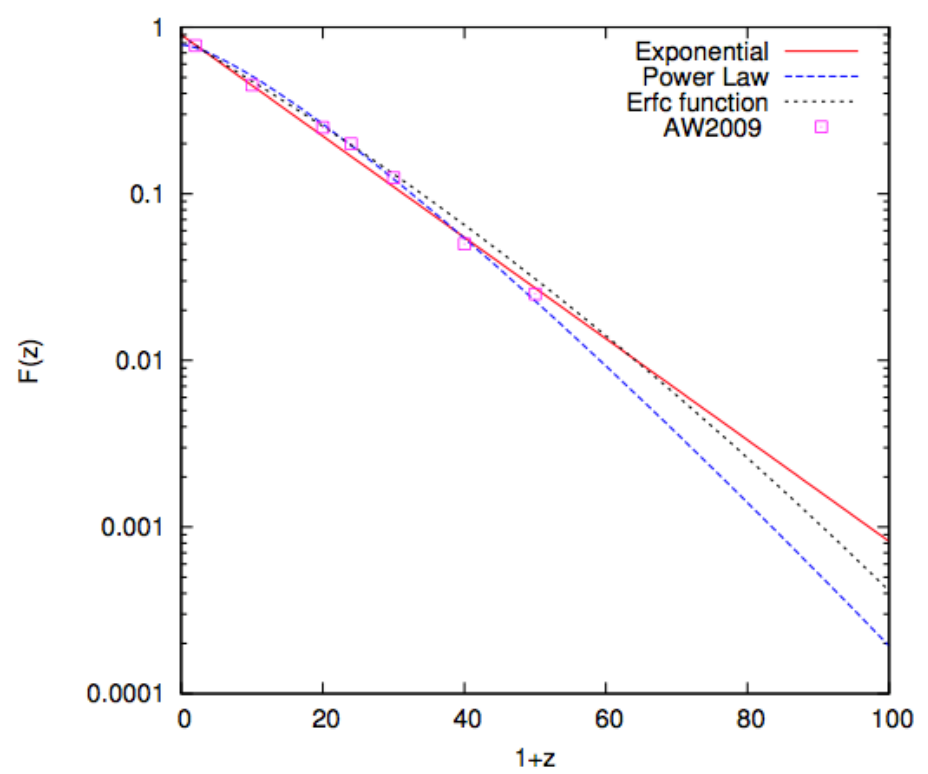

Figure 1. The three fitting functions $F(z)$ displayed together with N-body halo theory results from Padmanabhan (1991) (AW2009).

The three fitting functions are the single parameter power law and exponential models, and a two-parameter erfc function defined via the equations, 


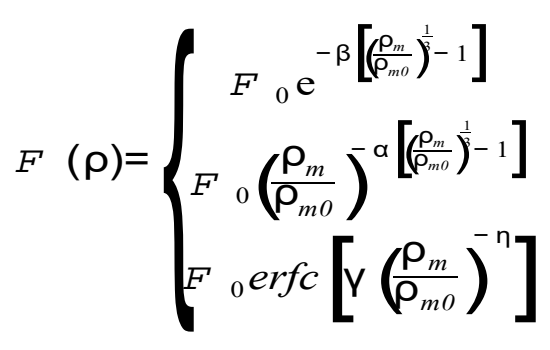

where $\alpha, \beta \gamma$ and $\eta$ are free parameters.

Now, if we consider the case for the power law, then substituting (15) into (5) and defining the energy density parameters,

$$
\Omega_{A}=\frac{8 \pi G a^{2} \rho_{A}}{3 H^{2}}
$$

and the dimensionless Hubble rate,

$$
E=\frac{H}{H_{0}}
$$

Where the Hubble parameter, $\mathrm{H}=\mathrm{Ha}^{-1}$ yields the ordinary differential equations describing the background interaction to be,

$$
\begin{aligned}
& \frac{d \Omega_{h}}{d a}=\frac{3}{a^{2}}(1-a-\ln a) \Omega_{h}+\frac{3}{2 \mathrm{a}}\left(\Omega_{M}-1\right) \Omega_{h} \\
& \frac{d \Omega_{f}}{d a}=-\frac{3}{a^{2}}(1-a-\ln a) \Omega_{h}+\frac{3}{2 \mathrm{a}}\left(\Omega_{M}-1\right) \Omega_{f}
\end{aligned}
$$

where we have used the definition of the conformal time,

$$
{ }^{\prime}=\frac{d}{d \eta}=a H \frac{d}{d a}
$$

The set of equations (18) can be closed by the dimensionless ODE for the Hubble parameter, 


$$
\frac{d E}{d a}=-\frac{3}{2 \mathrm{a}} E \Omega_{M}
$$

Figure 2 shows the effect of the interaction on the background density evolution for the case when $F$ is power law.

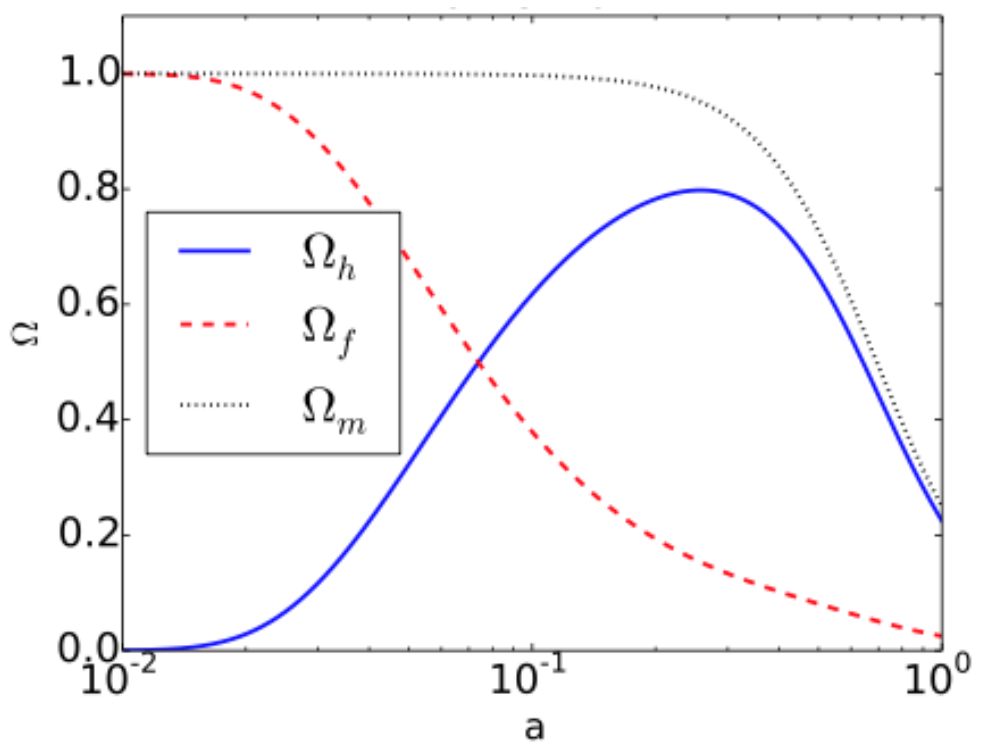

Figure 2. Evolution of the background energy densities displayed together with the total energy density.

The results show that at early times at the coupling epoch most of the matter is located outside halos and equal to the total matter in the universe. After the onset of structure formation the halos grow in size and accrete more matter so that the "free" matter outside the halos decreases. This happens until dark energy starts to dominate after which slows down the clustering of matter leading to a drop in the halo mass fraction and a corresponding less steeper decrease in the free matter as displayed by the blue and red-dashed curves in figure 3 respectively. 


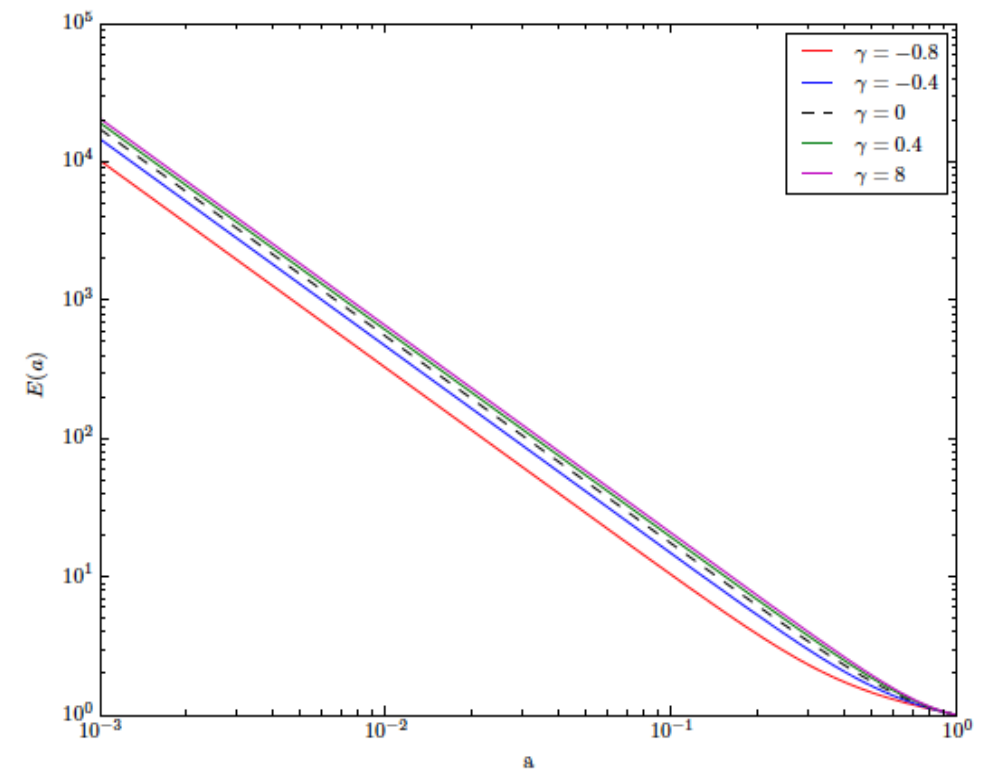

Figure 3. Evolution of the dimensionless Hubble expansion parameter for various interaction strengths. The black dashed line corresponds to the standard non-interacting case while $|\gamma|=Q\left(H_{0} \rho\right)^{-1}$ is a measure of the interaction strength.

\subsection{The perturbations}

For the perturbations, equation (3) yields analytical expressions for the A-fluid density contrasts to be

$$
\begin{aligned}
& \delta_{h}=\left(1+\rho_{m} \frac{\partial \ln F}{\partial \rho_{m}}\right)_{M} \\
& \delta_{f}=\left(1-\rho_{m} \frac{\Omega_{h}}{\Omega_{f}} \frac{\partial \ln F}{\partial \rho_{m}}\right) \bar{p}_{M}
\end{aligned}
$$

Hence for the power law model, (21) gives

$$
\begin{gathered}
\delta_{h}=\left[1-\frac{\alpha}{a}(1-\ln a-a)\right] \delta_{M} \\
\delta_{f}=\left[1+\frac{\alpha}{a} \frac{\Omega_{h}}{\Omega_{f}}(1-\ln a-a)\right] \delta_{M}
\end{gathered}
$$

To obtain a solution to the perturbation problem one only requires to 
solve the background equations (18) and (20) and the total matter density contrast evolution equation,

$$
\delta^{\prime}{ }_{M}=k^{2} v_{M}+3 \phi^{\prime}
$$

Which substituting (14) and using (19) gives the ODE

$$
\frac{d \delta_{M}}{d a}=\left(\frac{l^{2}}{a^{2} E \Omega_{M}}-\frac{9}{2} E\right) 2_{M} u_{M}-\frac{3}{a} \phi
$$

And to close the set of our ODE's we require the equations

$$
\frac{d \phi}{d a}=-\frac{3}{2} E \Omega_{M} u_{M}-\frac{\phi}{a}
$$

and

$$
\frac{d u_{M}}{d a}=-\frac{u_{M}}{a}-\frac{\phi}{a^{2} E}
$$

In order to solve the ODE's (24)-(26) we require initial conditions for $\delta_{M}$, and $v_{M}$ at decoupling. We obtain these as follows:

For $\phi$ we use the value of the gravitational scalar potential from inflation (Dodelson, 2003),

$$
\phi_{d}=\frac{9}{10} T(k) P_{\phi i n f}
$$

where $T(k)$ is the transfer function of the gravitational between the radiation domination era to the matter domination regime, and $P_{\text {ginf }}$ is the primordial power spectrum from inflation.

For $u_{M}$ we use the condition $\phi^{\prime}=0$ at decoupling to obtain, 


$$
u_{M}=-\frac{2 \phi}{3 \mathrm{H} \Omega_{\mathrm{M}}}
$$

For $\delta_{M}$ we use the relativistic Poisson equation,

$$
\nabla^{2} \phi=4 \pi G a^{2}\left(\delta_{M}-3 a E u_{M}\right) \Omega_{M}
$$

which gives,

$$
\delta_{M, d}=-\frac{k^{2} \phi}{4 \pi G a^{2} \Omega_{M}}+3 \mathrm{aE} u_{M}
$$

Figure 4 shows plots showing the large-scale evolution of the A-fluid density contrasts normalised to the total matter density contrast. The results show that at early times when most of the matter lies outside halos, the density perturbations in the free matter grow as those in the total matter while the energy transfer from the free fluid to the halo fluid damps any perturbations in the halo fluid. This continues until the gravity of the halos becomes large enough to counter the energy transfer damping. At this point the halo density perturbations start to grow again approaching the total matter density perturbations near today. This is consistent with the current picture since most of the matter observed in the universe today resides inside halos. The results show that in general, the halo fluid density perturbations are more sensitive to the interaction compared to the free fluid thus showing that such an interaction could bear significant effects on structure formation. 


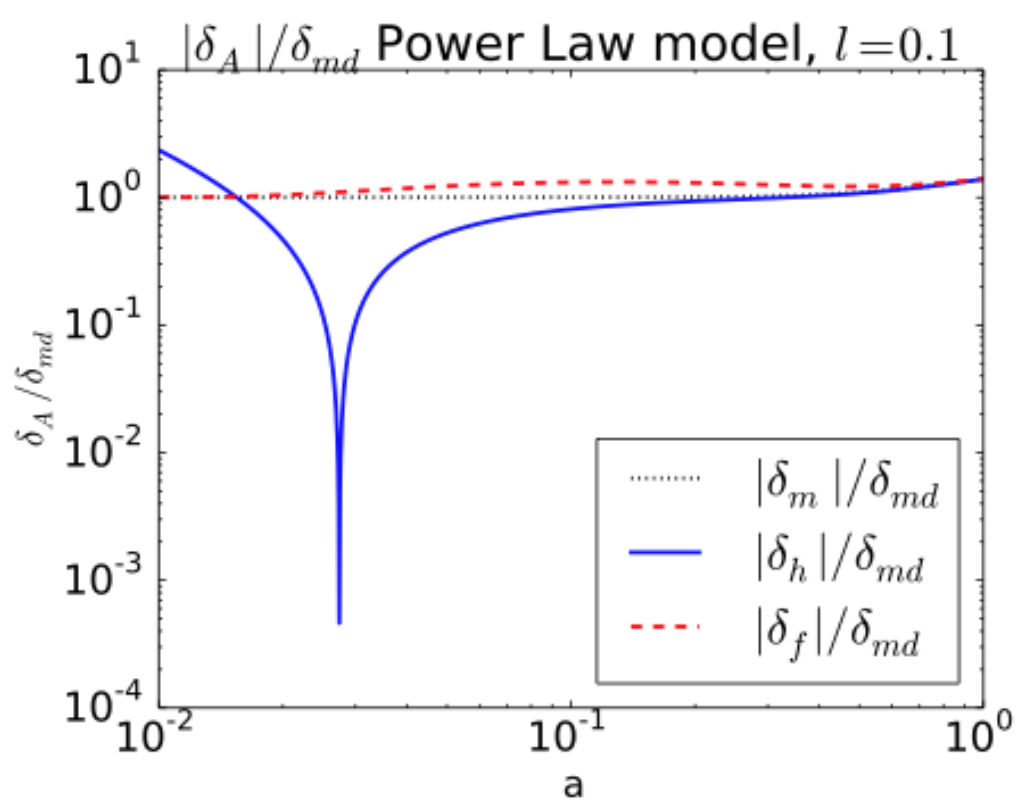

Figure 4. Evolution of the A-fluid density perturbations normalized to the total density contrast at decoupling. Here $l=k H_{0}^{-1}$ is the dimensionless wave vector.

\section{Discussion and Conclusions}

We have presented results from a new framework in which the matter fluid is split into two components, one accreting matter from the other thus giving rise to both energy and momentum transfer. We have shown that, in the background universe where the momentum transfer vanishes, the transfer of energy between the two components leads to matter density profiles that deviates from the standard noninteracting case. Since the halo density approaches the total matter density near today, our results show that the effects from the exchange of energy between the two matter components are small hence our background model can be considered to be an approximation of the standard $\Lambda \mathrm{CDM}$ model.

However, this is not exactly the case in the perturbed universe. The halo density perturbations are highly sensitive to the energymomentum transfer which causes damping of the perturbations at early times until gravity becomes large enough after which point the perturbations grow, approaching the standard $\Lambda$ CDM case. Since it is the halo fluid that plays a significant role in galaxy formation, the high sensitivity of the halo accretion reinforces the delicate nature of structure formation studies. More work needs to be done to confirm our results. 


\section{Acknowledgement}

GO acknowledges the organisers of the $4^{\text {th }}$ East African Astronomy Workshop in Kigali $31^{\text {st }}$ June- $4^{\text {th }}$ July 2014 for giving an opportunity for part of this work to be presented.

\section{References}

Angulo R. E. and White D.M. (2009). The Birth and Growth of Neutralino Haloes. Mon. Not. R. Astron. Soc., 000, 1-8.

Clemsonn T., Koyama K., Zhao G., Maartens R. and Valviita J. (2012). Interacting Dark Energy - constraints and degeneracies. Phys. Rev. D. 85, 043007 (2012).

Dodelson S. (2003). Modern Cosmology. Academic Press.

Durrer R. and Maartens R. (2008). Dark Energy and Dark Gravity. Gen. Rel. Grav. 85, 301-328 (2008

Padmanabhan T. (1993). Structure formation in the universe. Cambridge Univ. Press.

Planck Collaboration XVI (2014), Planck 2013 results. XVI. Cosmological parameters, $A \& A, 571, \mathrm{~A} 16$, arXiv: 1303.5076.

Valviita J, Majerotto E. and Maartens R. (2008). Large-scale instability in interacting dark energy and dark matter fluids. JCAP, 0807, 020. 